\title{
A Dual Origin for Bcr-Abl Gene Translocation/Fusion as Dynamics of Synergism of the Hematopoietic Stem Cell and Hemangioblast in Chronic Myeloid Leukemia
}

\section{Lawrence M. Agius*}

Department of Pathology, Mater Dei Hospital, Tal-Qroqq, University of Malta Medical School, Msida, Malta Europe

${ }^{*}$ Corresponding author: Lawrence M. Agius, Department of Pathology, Mater Dei Hospital, Tal-Qroqq, University of Malta Medical School, Msida, Malta Europe, Tel: 356-21451752; E-mail: lawrence.agius@um.edu.mt

Received date: December 14, 2015; Accepted date: December 29, 2015; Published date: December 30, 2015

Copyright: (C) 2015 Agius LM. This is an open-access article distributed under the terms of the Creative Commons Attribution License, which permits unrestricted use, distribution, and reproduction in any medium, provided the original author and source are credited.

\begin{abstract}
Contextual BCR-ABL tyrosine kinase over-activity determines in formulated fashion the emergence of proliferation and anti-apoptosis that arise largely as derived phenomena of otherwise homeostatic mechanisms of the c-ABL gene within hematopoietic stem cells and hemangioblasts in the bone marrow. The ability to suppress almost completely, both in terms of phenotype and cytogenetically, the myeloid cell line expansion by imatinib mesylate is indicative of a phenomenon that depends strictly on the transformed status of the cell of origin in the chronic myeloid leukemia process. It is with relevance to complex participation of the dynamics of the fused BCR$\mathrm{ABL}$ protein product that contextual conditioning of the cells of origin of the gene translocation further motivates the dimensional expansion of the transformed myeloid cell clones to increasing proliferative rates, thus leading to blast crisis as eventual loss of differentiating potential.
\end{abstract}

Keywords: BCR-ABL; Hematopoietic stem cell; Hemangioblast; Leukemia

\section{Introduction}

The targeting of the hematopoietic stem cells bearing BCR-ABL translocation fusion gene is sufficient for phenotypic control of the proliferating myeloid cells in patients suffering from the chronic phase of chronic myeloid leukemia. Noncoding RNAs are involved in CML in particular, by regulating gene expression through binding to target mRNAs [1]. The dimensions of such suppression, however, allow for persistence of non-replicating hematopoietic stem cells in patients treated with imatinib mesylate. Imatinib has potent anti-cancer effect against leukemia associated with BCR-ABL and with solid tumors over-expressing c-kit and Platelet Derived Growth Factor Receptor [2]. Within such scenario, the question arises whether hemangioblasts are a real primary target for BCR-ABL fusion translocation; such possibility is significant in the possible realization of involvement of both hematopoietic stem cells and endothelial cells in malignant transformation, as has also been suggested in breast carcinoma and glioblastoma lesions.

\section{Primitive Cell of Origin}

Such considerations indicate the directive involvement of a primitive cell line that is primarily non-differentiated but capable of significant divergent potential for differentiation as multi-potent cells of involvement in the transformation process. S-phase progression of the cell-cycle may be inhibited against lymphoid crisis in CML and Philadelphia chromosome-positive acute lymphoblastic leukemia [3].

The inclusive phenomenon of malignant transformation in the initial stages of chronic myeloid leukemia is derived from considerations also of the quiescent nature of such cells that, to a significant degree, prove to be elements of diversity in the dual process of transformation and of mitogenesis of the targeted cells.

\section{Chronic Phase}

In line with the development of the chronic phase of chronic myeloid leukemia, there arises a complex derivation of the BCR-ABL fusion gene that is cytogenetically recognizable as the Philadelphia chromosome $[\mathrm{Ph}]$. In $98 \%$ of CML patients with the $\mathrm{Ph}$ chromosome, there develops cleavage of the BCR gene within its major breakpoint region and breakage of the ABL within a 100-kbp region of exon 2a [4]. The intricate definition of the nature of the constitutive activation status of the tyrosine kinase domain of the ABL gene is linked to an overlapping break point of the BCR gene in the process of reciprocal translocation. Region choice for canonical siRNA design algorithms is restricted in a fusion junction site as BCR-ABL but potency may be enhanced by optimization outside of target sequence [5]. The mechanics of the translocation process itself between $\mathrm{ABL}$ and $\mathrm{BCR}$ appear obscure but might be implicated within the general presence of BCR-ABL fusion gene even in healthy individuals, albeit at low levels.

The discovery of oncogenic drives and development of targeted therapies such as imatinib has markedly improved outcome in CML patients [6]. Cancer cells efficiently utilize limited energy sources, and drugs that target cellular metabolism further improve the efficacy of imatinib therapy [7].

Such presence in patients, who do not in fact constitute early stages of chronic myeloid leukemia indicates a possible emergence of normal physiologic roles of $\mathrm{ABL}$, within normal contextual conditioning of hematopoietic cells. In such manner, c-ABL is abundant especially within the cytosol and is present also combined with nuclear chromatin. 
The BCR-ABL fusion protein product appears therefore to actively constitute an essentially over-amplified consequential process that is beyond simple considerations of parametric homeostatic control. Metastasis suppressor 1 is downregulated in hematopoietic stem and progenitor cells in patients with CML [8]. It is such quantitative overexpression of the tyrosine kinase activity that would promote a constitutive potentiality for expression of such tyrosine kinase. Also, variant complex translocations involving a third chromosome may be present in 5-8\% of CML patients [9].

Significant is the emergence of the p120 BCR-ABL fusion protein as the common isoform in the development of growth-factor independence of the malignant myeloid cell proliferation in CML patients. By contrasting the conformational free energy landscape of mutants resistant to imatinib action with those of the wild-type tyrosine kinases it is possible to better understand the emergence of drug resistance [10].

\section{Phenotype}

Consideration of the chronicity of the transformed myeloid cells is relative to advanced stages of the disease where blast crisis emerges in terms that phenotypically are expressed by both high proliferative rates and also as essential non-differentiation status; such blast cells are reflected in the generation of a large range of additional genetic lesions and also in the insensitivity of the tyrosine kinase activity to suppression by imatinib mesylate. Proteolysis Targeting Chimera technology utilizes small molecules that recruit target proteins such as $\mathrm{BCR}-\mathrm{ABL}$ and $\mathrm{c}-\mathrm{ABL}$ for the ubiquitination-proteosome for degradation [11].

It would appear that translocation in the generation of the BCR$\mathrm{ABL}$ fusion gene occurs essentially within non-proliferative stem cells in a manner that is strictly cell-context dependent.

The realization of further expansion of the clonal proliferation of multi-potent cells is further indicative of possible primary targeting of the hemangioblast in a manner that bridges hemopoiesis and endothelial vascular genesis within the bone marrow, in early stages of transformation in CML patients. Mutations in chromatin regulators often result in more stem-like cells that seed a bulk leukemic population and therapeutic agents targeting these regulators are being developed [12].

\section{BCR-ABL Transformation}

The derivative dimensions included within the establishment status of the BCR-ABL translocation allow for a permissive production of the corresponding fusion protein that may exceed the controllable limit of action of the highest tolerable levels achieved by imatinib mesylate therapy. Hyaluronan oligomers sensitize CML cells to the antiproliferative effect of imatinib by increasing apoptosis and senescence [13].

The mechanism and natural history of CML is still unclear [14]. Hence, it is significant to view the $\mathrm{c}-\mathrm{ABL}$ as potential overlap phenomenon with the BCR-ABL fusion gene in determining the nature of the malignant transformation process within hematopoietic stem cell and hemangioblast as a combined integral phenomenon of synergistic enhancement.

The further compounding effects of such synergism are reflected in a dual population of cell types that ensure the promoted transformation event as essential establishment and maintenance of the tyrosine kinase over-activity in terms especially of constitutive activation. There is a significant association of defective apoptosis arising from promoter polymorphisms of Fas-FasL genes with the development of CML [15].

\section{Experimental Transfer}

Biologic ineffectiveness in transfer of malignant transformation to many cell lines and to murine models indicates the failed susceptibility of a number of cell types to the establishment of early stages of oncogenesis in CML, in vitro and in vivo. The lack of a faithful model of the chronic phase of CML in the laboratory testifies to poorly understood dynamics of the translocation/fusion phenomenon in genesis of the BCR-ABL gene complex, and especially to the breakpoint dynamics of both $\mathrm{BCR}$ and $\mathrm{ABL}$, but particularly within the breakage cluster regions of the $\mathrm{BCR}$ gene. In particular, the therapeutic resistance by the gatekeeper mutation T315I in BCR-ABL to imitinib is a significant problem clinically [16], but pyrazolopyrimides may serve as lead compounds for development. MicroRNA employing non-viral vectors have been utilized to inhibit various cancer cells, and may prove useful in CML hematopoietic stem cells [17].

\section{Hemangioblast/Hematopoietic Stem Cell}

$\mathrm{ABL}$ has been recognized as essential for the transformation process whereas the BCR appears to contribute to the maintenance of establishment and proliferation of the proliferating clone expansion in CML. Such considerations indicate the emergence of individual gene effects within complex realization of effects in oncogenesis. Modulation of aging-related inflammation within the bone marrow micro-environment may enhance the emergence of oncogenic events in stem cells [18]. Isohiocyanate treatment may correct inflammatory and defective DNA repair mechanisms in hematopoietic tissues [19]. The development of effective contextual requirement for the specific cellular micro-environment is dependent on the active genesis of the translocation event itself, but this also promotes the participation of the possible dual relevance of the hemangioblast and hematopoietic stem cell in malignant transformation.

Dysregulated cholesterol metabolism and/or accumulation occur in leukemia, and 2-hydroxypropyl-beta-cyclodextrin shows anti-CML effects by modifying cholesterol [20].

The pool of quiescent hematopoietic stem cells and the further possible forms of co-operability of hemangioblasts in malignant transformation indicate a basic dualism that is further defining dimension for contextual cellular requirements in the oncogenesis of CML. Dasatinib is a novel, multi-targeting kinase inhibitor of breakpoint cluster region Abelson [BCR-ABL] and Src family kinases, with a time-dependent anti-leukemic effect [21].

Parameters of multi-potentiality of these cells allow for a permissiveness of excessive proliferative cells that progress in terms of added realized constitutive activation of the tyrosine kinase of the fused ABL. The development of selective kinase inhibitors is challenging due to the large size of the kinome and the structural similarity between kinase active sites [22]. Multi-targeting of both BCR-ABL kinase and microtubules may be achieved with 2acylaminothiophene-3-carboxamides [23]. 


\section{Genetic Instability}

Dimensional correlation allows for the recognition of malignant permissiveness that correlates with possible generation of genetic instability, and thus to the generation of accumulated genetic defects within the neoplastic cells. Under-expression of miR-130a functions as tumor suppressor by inhibiting multiple anti-apoptosis proteins including BCL-2 [24]. Added to such phenomenon, however, is the significant phenotypic suppression of the malignant myeloid cells by primarily targeting the cells of origin of the chronic phase of CML. More inhibitors of the mutated ABL tyrosine kinase are absolutely required [25]. Inhibitors of BCR-ABL tyrosine kinase kill CML cells by stimulating pro-apoptotic BIM and inhibiting anti-apoptotic BCL2, BCLxL and MCL1 [26].

\section{Diversity in Transformation}

Diversity of potential transformation is hence linked to the multipotentiality of the cancer stem cell concept for oncogenesis but this is itself conditioned by dimensions found within the microenvironment of the bone marrow.

Such conditioning appears linked to the homeostatic functions of the c-ABL gene in enhancing intercellular adhesion and communication, on the one hand, and also of the determined cellular morphology of the targeted cells. Indicative parameters include integrin signaling and also a binding of the $\mathrm{ABL}$ to the actin within the cytosol of the cells concerned. A cytosol-nuclear transport also is relevant in that the BCR-ABL fusion protein loses nuclear target potential. Hence the relevance of strict dynamics of the BCR-ABL fusion protein is critical in the ongoing process in CML oncogenesis that is accompanied by a wide variety of down-stream signaling pathways such as Jun, STAT5, Ras pathway. Included also is the implied dysfunction of the $20 \mathrm{~S}$ proteasome-ubiquitin mechanisms. Better understanding of CML hematopoietic stem cell biology is required to eradicate these cells responsible for disease recurrence and of residual disease [27].

\section{Alternative Pathways and Blast Crisis}

CML therefore emerges as a series of alternative pathways of proliferation, on the one hand, and of differentiation pathways that are operative within the pool of differentiated malignant myeloid cells in patients who enter the chronic phase of the CML. Highly significant is the further advanced disease-state where differentiating potential is lost within contextual conditioning in establishment of higher proliferative rates of the malignant cells.

The BCR-ABL fusion gene doubles the expression level of genes involved in the regulation of the cell cycle, proliferation and apoptosis of CD34+ cells, with implications also of modified genes in PI3K/AKT and MAPK signaling in CML patients [28].

The blast crisis in CML patients indicates a dynamic process of evolving oncogenesis that is inclusive phenomenon for participation of the accumulating genetic lesions within the transformed myeloid cells. Linked to such phenomenon is the emergence in some patients of the p190 isoform of the BCR-ABL translocation fusion gene in patients with the acute ALL phenotype lacking an initial chronic phase.

Overall, there develops a progression of the disease state that closely correlates with dynamics of establishment of the malignant process that is maintained by a BCR-ABL fusion gene. The significant suppression possible by specific imatinib-targeting of the cell of origin of the malignant transformation of CML indicates the highly constitutive nature of a malignant change that is ABL-tyrosine-kinase mediated.

\section{Concluding Remarks}

Permutation of dynamics of the BCR-ABL fusion protein lies at the center of operative transfer of the constitutive activation of the tyrosine kinase domain of the ABL protein. Added formulation of breakage point involvement of the BCR and of significant participation in establishment of the malignant phenotype within the contextual cellular series of dual hematopoietic stem cell and of hemangioblast would lead to the recognition of a micro-environmental series of transforming changes inducing proliferation and anti-apoptosis leading to the eventual creation of blast cells that do not differentiate.

Further to the systematic parameters of proliferation is added an overall projected loss of potential differentiation that is maintained at the expense progressively increasing proliferative activity.

\section{References}

1. Wang X, Chen K, Guo G, Chen JL (2015) Noncoding RNAs and their functional involvement in regulation of chronic myeloid leukemia. Brief Funct Genomics .

2. Wei Y, To KK, Au-Yeung SC (2015) Synergistic cytotoxicity from combination of imatinib and platinum-based anticancer drugs specifically to Bcr-Abl positive leukemia cells. J Pharmacol Sci pii: S1347-8613(15)002190-4.

3. Nemoto A, Saida S, Kato I, Kikuchi J, Furukawa Y, et al. (2015) Specific anti-leukemic activity of PD0332991, a CDK4/6 inhibitor, against Philadelphia-chromosome positive lymphoid leukemia. Mol Cancer Ther 15: 94-105.

4. Lund HL, Hughesman CB, McNeil K, Clemens S, Hocken K, et al. (2015) Initial diagnosis of chronic myelogenous leukemia based on quantification of M-BCR status using droplet digital PCR. Anal Bioanal Chem.

5. Gavrilov K, Seo YE, Tietjen GT, Cui J, Cheng CJ, et al. (2015) Enhancing potency of siRNA targeting fusion genes by optimization outside of target sequence. Proc Natl Acad Sci U S A 112: E6597-6605.

6. Redmond KL, Papafili A, Lawler M, Van Schaeybroeck S (2015) Overcoming Resistance to Targeted Therapies in Cancer. Semin Oncol 42: 896-908.

7. Shinohara H, Kumazaki M, Minami Y, Ito Y, Sugito N, et al. (2016) Perturbation of energy metabolism by fatty-acid derivative AIC-47 and imatinib in BCR-ABL-harboring leukemic cells. Cancer Lett 371: 1-11.

8. Schemionek M, Herrmann O, Merle Reher M, Chatain N, Schubert C, et al. (2015) MTSS1 is a critical epigenetically regulated tumor suppressor in CML. Leukemia .

9. Asif M, Hussain A, Malik A, Rasool M (2015) Three-way complex variant translocation involving short arm chromosome $(1 ; 9 ; 22)(\mathrm{p} 36 ; \mathrm{q} 34 ; \mathrm{q} 11)$ in a chronic myeloid leukemia patient. Oncol Lett 10: 1628-1730.

10. Lovera S, Morando M, Pucheta-Martinez E, Martinez-Torrecuadrada JL, Saladino G, et al. (2015) Towards a molecular understanding of the link between Imatinib resistance and kinase conformational dynamics. PLoS Comput Biol 11: e1004578.

11. Lai AC, Toure M, Hellerschmied D, Salami J, Jaime-Figueroa S, et al. (2015) Modular PROTAC Design for the Degradation of Oncogenic BCR-ABL. Angew Chem Int Ed Engl. 55: 807-810.

12. Shingleton JR, Hemann MT (2015) The Chromatin Regulator CHD8 Is a Context-Dependent Mediator of Cell Survival in Murine Hematopoietic Malignancies. PLoS One 10: e0143275. 
Citation: Agius LM (2015) A Dual Origin for Bcr-Abl Gene Translocation/Fusion as Dynamics of Synergism of the Hematopoietic Stem Cell and Hemangioblast in Chronic Myeloid Leukemia. J Leuk 3: 203. doi:10.4172/2329-6917.1000203

Page 4 of 4

13. Lompardía SL, Díaz M, Papademetrio DL, Mascaró M, Pibuel M, et al. (2015) Hyaluronan oligomers sensitize chronic myeloid leukemia cell lines to the effect of Imatinib. Glycobiology.

14. Ma W, Ma N, Chen X, Zhang Y, Zhang W (2015) An overview of chronic myeloid leukemia and its animal models. Sci China Life Sci 58 1202-1208.

15. Edathara PM, Gorre M, Kagita S, Vuree S, Cingeetham A, et al. (2015) Association of promoter polymorphisms of Fas -FasL genes with development of Chronic Myeloid Leukemia. Tumour Biol.

16. Liu X, Kung A, Malinoski B, Prakash GK, Zhang C, et al. (2015) Development of Alkyne-Containing Pyrazolopyrimidines To Overcome Drug Resistance of Bcr-Abl Kinase. J Med Chem 58: 9228-9237.

17. Ma W, Liu J, Xie J, Zhang X, Zhou H, et al. (2015) Modulating the growth and imatinib sensitivity of chronic myeloid leukemia stem/progenitor cells with Pullulan/MicroRNA nanoparticles in vitro. J Biomed Nanotechnol 11: 1961-1974.

18. Henry CJ, Casas-Selves M, Kim J, Zaberezhnyy V, Aghili L, et al. (2015) Aging-associated inflammation promotes selection for adaptive oncogenic events in B cell progenitors. J Clin Invest 125: 4666-4680.

19. Lawson AP, Long MJ, Coffey RT, Qian Y, Weerapana E, et al. (2015) Naturally occurring isothiocyanates exert anticancer effects by inhibiting deubiquitinating enzymes. Cancer Res 75: 5130-5142.

20. Yokoo M, Kubota Y, Motoyama K, Higashi T, Taniyoshi M et al. (2015) 2Hydroxypropyl- $\hat{I}^{2}$-Cyclodextrin Acts as a Novel Anticancer Agent. PLoS One 10: e0141946.

21. Ishida Y, Murai K, Yamaguchi K, Miyagishima T, Shindo M, et al. (2015) Pharmacokinetics and pharmacodynamics of dasatinib in the chronic phase of newly diagnosed chronic myeloid leukemia. Eur J Clin Pharmacol.

22. Gower CM, Thomas JR, Harrington E, Murphy J, Chang ME, et al. (2015) Conversion of a single polypharmacological agent into selective bivalent inhibitors of intracellular kinase activity. ACS Chem Biol.

23. Cao R, Wang Y, Huang N (2015) Discovery of 2-Acylaminothiophene-3Carboxamides as Multitarget Inhibitors for BCR-ABL Kinase and Microtubules. J Chem Inf Model 55: 2435-2442.

24. Zhu X, Zhao H, Lin Z, Zhang G (2015) Functional studies of miR-130a on the inhibitory pathways of apoptosis in patients with chronic myeloid leukemia. Cancer Gene Ther 22: 573-580.

25. Kumar H, Raj U, Gupta S, Varadwaj PK (2015) In-silico identification of inhibitors against mutated BCR-ABL protein of chronic myeloid leukemia: a virtual screening and molecular dynamics simulation study" J Biomol Struct Dyn 19: 1-34.

26. Ishii Y, Nhiayi MK, Tse E, Cheng J, Massimino M, et al. (2015) Knockout Serum Replacement Promotes Cell Survival by Preventing BIM from Inducing Mitochondrial Cytochrome C Release. PLoS One 10: e0140585.

27. Wang Z, Chen CC, Chen W (2015) CD150- Side Population Defines Leukemia Stem Cells in a BALB/c Mouse Model of CML and Is Depleted by Genetic Loss of SIRT1. Stem Cells .

28. ÄEokiÄ VP, MojsiloviÄ S, JaukoviÄ A, Kraguljac-KurtoviÄ N, MojsiloviÄ S, et al. (2015) Gene expression profile of circulating CD34(+) cells and granulocytes in chronic myeloid leukemia. Blood Cells Mol Dis 55: $373-381$. 